\title{
\begin{tabular}{|l|l|l|l|l|}
\hline $\mathrm{M}$ & $\mathrm{R}$ & $\mathrm{S}$ & Internet Journal of & Nitride Semiconductor Research \\
\hline
\end{tabular}
}

Volume 1, Article 14

\section{Bandgap Variation at the Isostructural Phase Transformation of Wurtzite $\ln \mathbf{N}$}

\author{
L. Bellaiche \\ National Renewable Energy Laboratory \\ K. Kunc, M. Besson \\ CNRS and Université P. and M. Curie
}

This article was received on June 3, 1996 and accepted on October 7, 1996.

\begin{abstract}
The pressure variation of the bandgap, at the isostructural phase transition of wurtzite $\ln N$, is determined theoretically, using the first-principles total-energy pseudopotential method. It is found that the bandgap (as well as the structural parameters) exhibit three different types of behavior, in three regions of pressure. Optical experiments at low temperatures could then be employed to directly identify the two different wurtzite phases of InN.
\end{abstract}

By using the first-principles total-energy pseudopotential method, we recently studied [1] the behavior of wurtzite InN under hydrostatic pressure. All the structural parameters were relaxed in [1], at all volumes considered, and the relative variations of the volume, of the lattice constant, and of the c/a axial ratio were found to be in remarkable agreement with experiment [2], over the whole range of the measured pressures (see Figure 1). These calculations point to the existence of a second order isostructural phase transition which occurs in the same pressure range as the wurtzite-rocksalt first-order phase transformation, i.e. between 12 and $15.5 \mathrm{GPa}$. This isostructural phase transition completely changes the behavior of the structural parameters, as can be seen from Figure 1 . For instance, it induces a sharp decrease of the axial ratio c/a and an increase of the internal parameter $u$, which indicates that the In and $\mathrm{N}$ sublattices slide relative to each other until a new stable position is reached around $16 \mathrm{GPa}$. In this respect, InN behaves quite differently from $\mathrm{GaN}$, for which c/a is nearly independent of pressure [2]. Since it was shown in Ref. [1] that a specific second-neighbor interaction between indium atoms was instrumental in the isostructural phase transition process, the difference of behavior between InN and GaN can be related to the difference of the cation atomic sizes between the two nitrides.

We also proposed in [1] that this new phase transition, which still awaits experimental observation, can be considered as a pretransitional effect announcing the reconstructive first order transition. The existence of pretransitional phenomena is not unknown to solid state physics, but these effects have been so far established in rather few semiconductors. For instance, in the mercury chalcogenides $\mathrm{HgSe}$ and $\mathrm{HgTe}$ [3] (which are tetrahedrally coordinated, too), the first order transition from the zincblende to the cinnabar structure is preceded by a strongly non-linear behavior of the elastic constants, leading to their fast decrease with pressure, just prior to the transition. Pretransitional effects have also been proposed in Ref. [4], as an explanation of the Si and InSb diffraction patterns recorded, as well, just prior to the high-pressure transition.

The aim of the present contribution is to investigate the behavior of the bandgap of $\operatorname{InN}$ with pressure, in order to find out whether the instability found in InN prior to its transition to the high-pressure phase, instead of being observed by X-ray methods, could also be detected by optical transitions. For this purpose we are using the density-functional theory in the Local Density Approximation (LDA) [5], within the plane-wave pseudopotential scheme, and in momentum space [6], for fixed ionic configurations, and in the same conditions as in our previous work, Ref. [1]. We employ the norm-conserving pseudopotentials of Ref. [7], and the Ceperley-Alder exchange and correlation [8] as parameterized by Perdew and Zunger [9]. A plane-wave cutoff of 70 Ry is used. The $\vec{k}$-space summation is performed (using the semiconductor sampling) with 7 'special points' which, in the Monkhorst-Pack notation [10], correspond to $\left(a_{1}, a_{2}, q_{3}\right)=(4,4,2)$. After the structural relaxations have been accomplished as in Ref. 
[1], all »residual“ Hellmann-Feynman forces are smaller than 0.002 millidynes.

The calculated bandgap $E_{g}$, which is direct, is shown in Figure 2. Since the semiconductor bandgaps derived from the LDA eigenvalues are too small, we displayed our results shifted by a »scissor operator", viz. we added to the LDA bandgap a constant term independent of pressure. By this correction we expect to obtain realistic gaps $\mathrm{E}_{\mathrm{g}}$ at all pressures, since it has been demonstrated [11] that even if the LDA underestimates the gaps, it leads to correct predictions for their pressure variation (i.e for the linear pressure coefficients). The added term was chosen as the difference between the $0 \mathrm{~K}$-experimental and the calculated $(0.08 \mathrm{eV})$ bandgaps at equilibrium; the 0 $\mathrm{K}$-experimental value of $1.94 \mathrm{eV}$ for the bandgap was derived from the experimental value at $300 \mathrm{~K}(1.89 \mathrm{eV}$ [12]) by correcting it with the bandgap temperature coefficient of $-1.8 \times 10^{-4} \mathrm{eV} / \mathrm{K}[12]$.

The bandgap variation spans the visible region of spectrum. We can observe from Figure 2 that, up to $\approx 12 \mathrm{GPa}$, the bandgap increases, nearly linearly, with pressure. This linear dependence of the bandgap on pressure is described by the coefficient $\mathrm{dE}_{\mathrm{g}} / \mathrm{dp}$ of $28 \mathrm{meV} / \mathrm{GPa}$, which is in good agreement with both the experimental and other theoretical values (25-33 meV/GPa) [13] [14]. Then, above $12 \mathrm{GPa}$, the wurtzite bandgap changes its behavior, and remains quite constant, at about $2.35 \mathrm{eV}$, at the onset of the second order isostructural phase transition (cf. Figure 1).

Above $16 \mathrm{GPa}$, where the wurtzite structure has in actual experiments already completely disappeared (due to the first order phase transition to the rocksalt structure), the calculations for the (unstable) wurtzite structure show the usual linear pressure-variation of the bandgap, with a different pressure coefficient $\mathrm{dE}_{\mathrm{g}} / \mathrm{dp}=22 \mathrm{meV} / \mathrm{GPa}$.

Although the concurrent first-order transition to the rocksalt structure occurs in the intermediate region of pressures (12.1 GPa in the room-temperature-, upstroke measurements on powdered samples [2]), the two transformations still might be discerned in experiments at low temperature, and on a monocrystal. In this case the region of metastability of the low-pressure phase is expected to be enlarged (hysteresis), and the upstroke transition pressure to be shifted to a higher value. Then the isostructural (second-order) phase transition might set in before the actual transformation to rocksalt structure destroys the wurtzite lattice.

The behavior of the bandgap between 12 and $15.5 \mathrm{GPa}$ reflects the modifications of the electronic charge density which cause the isostructural phase transition. As a non-linear behavior of any of the structural parameters (i.e. of interatomic bonding) is correlated with a redistribution of the electronic charge density, it is straightforward to make a parallel between a "phase transition" within the electronic structure and the observed transformation of the atomic structure. In metals, such a relation (an "Electronic Topological Transition") was suggested recently [15] to explain the isostructural transformations in Cs (first order) and Zn (second order). In the present case, the $20 \%$ decrease of the pressure coefficient of the gap, which our calculations show to exist between the low- and high-pressure wurtzite structures, is a direct measure of the difference of interatomic bonds, that is reflected by the electronic density distributions in the two structures.

The bandgap variation shown in Figure 2 should be readily observed by optical experiments (e.g. absorption) which are easier to perform, and less demanding on the size of the samples, than X-ray measurements [2] of the variation of the structural parameters given in Figure 1. The two different wurtzite phases could then be directly evidenced by the different behavior of the bandgap under pressure.

The authors wish to thank S. Froyen, A. Zunger and C.-Y. Yeh for useful discussions. This work was partly supported by a NATO grant, and the computer resources were provided by the Scientific Committee of IDRIS (Institut du Développement et des Ressources en Informatique Scientifique), Orsay, France.

\section{References}

[1] L. Bellaiche, K. Kunc, J. M. Besson, unpublished (1996).

[2] Masaki Ueno, Minoru Yoshida, Akifumi Onodera, Osamu Shimomura, Kenichi Takemura, Phys. Rev. B 49, 14-21 (1994).

[3]A. J. Miller, G. A. Saunders and Y. K. Yogurtcu, "The pressure dependence of elastic constants and bond bending in HgTe", Phil. Mag. A 43, 1447 - 1471 (1981)

[4] D. R. Yoder-Short, R. Colella, B. A. Weinstein, Phys. Rev. Lett. 49, 1438-1441 (1982). 
[5] P. Hohenberg, W. Kohn, Phys. Rev. 136, B864-B871 (1964).

[6] J. Ihm, A. Zunger, M. L. Cohen, J. Phys. C 12, 4409-4422 (1979).

[7] G. B. Bachelet, D. R. Hamann, M. Schlüter, Phys. Rev. B 26, 4199-4228 (1982).

[8] D. M. Ceperley, B. J. Alder, Phys. Rev. Lett. 45, 566-569 (1980).

[9] J. Perdew, A. Zunger, Phys. Rev. B 23, 5048-5079 (1981).

[10] H. J. Monkhorst, J. D. Pack, Phys. Rev. B 13, 5188-5192 (1976).

[11] K. J. Chang, S. Froyen, M. L. Cohen, Sol. St. Comm. 50, 105-107 (1984).

[12] S. Strite, H. Morkoç, J. Vac. Sci. Technol. B 10, 1237-1266 (1992).

[13] JH Edgar, (Editor), Properties of Group III Nitrides (Electronic Materials Information Service (EMIS), London, 1994).

[14] N. E. Christensen , I. Gorczyca, Phys. Rev. B 50, 4397-4415 (1994).

[15]M. Steiner, W. Potzel, H. Karzel, M Kofferlein, G. M. Kalviu and P. Blaha, J. Phys. C 8, 3581 (1996) 


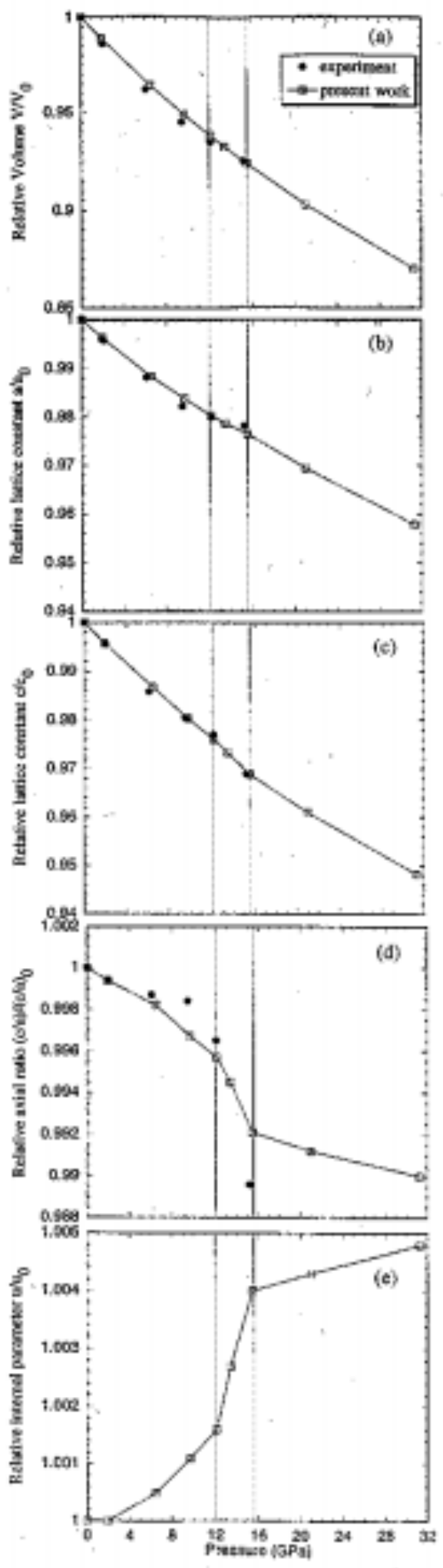

Figure 1. Relative variation of the structural parameters of wurtzite InN with pressure: (a) volume $\mathrm{V}$, (b) lattice constant a, (c) lattice constant c, (d) axial ratio c/a, and (e) internal parameter $u$. $v_{0}, a_{0}, c_{0},(c / a)_{0}$ and $u_{0}$ stand for the equilibrium values of the structural parameters, the solid line and empty squares are our calculations from Ref. [1], and the solid black circles are the experimental data [2]. The two vertical lines delimit the region of phase coexistence: at $12 \mathrm{GPa}$, the wurtzite lattice is unstable since it starts transforming to the $\mathrm{NaCl}$ structure, and at 15.5 $\mathrm{GPa}$, the transformation is completed. 


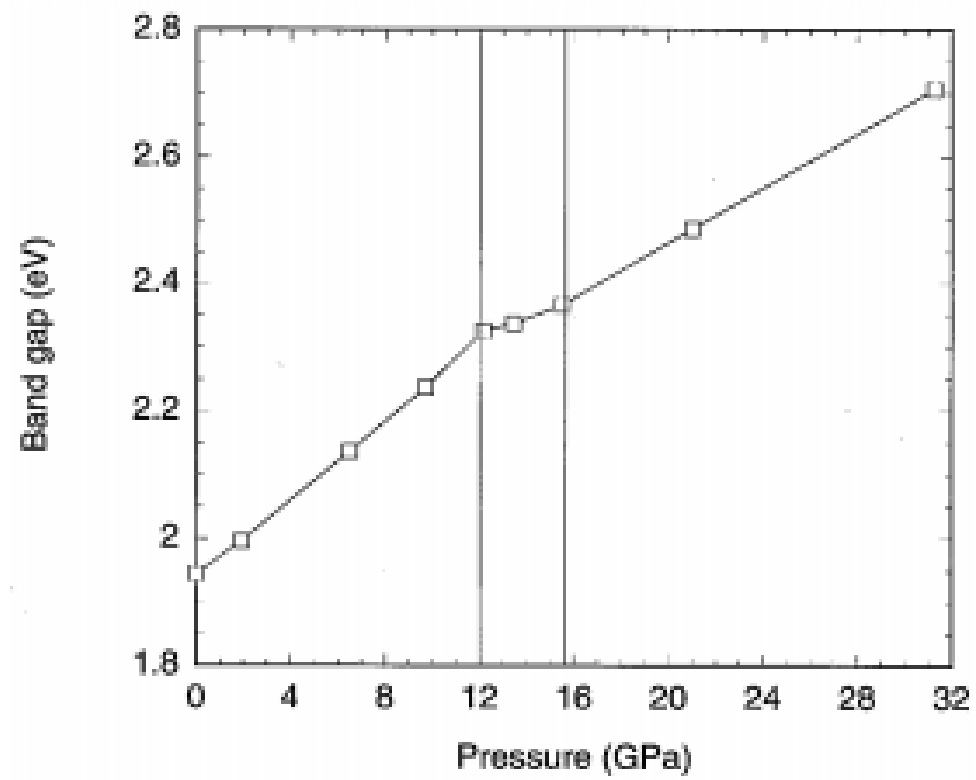

Figure 2. Relative variation of the calculated bandgap of wurtzite $\mathrm{InN}$ with pressure. The "scissor operator", independent of pressure, was applied to the LDA-calculated eigenvalues - see the text. The two vertical lines delimit the region of phase coexistence, as defined in Figure 1.

(C) 1996-1998 The Materials Research Society

\begin{tabular}{|l|l|l|l|l|}
\hline M & R & S & Internet Journal of & Nitride Semiconductor Research \\
\hline
\end{tabular}

